\title{
Heparin mimetic peptide nanofiber gel promotes regeneration of full thickness burn injury
}

\author{
Fatih Yergoz ${ }^{a}$, Nurcan Hastar ${ }^{a}$, Cagla Eren Cimenci ${ }^{a}$, Alper Devrim Ozkan ${ }^{a}$, \\ Turgay Tekinay $^{\text {b, c }}$, Mustafa O. Guler ${ }^{\text {a, e, }{ }^{* *}}$, Ayse B. Tekinay ${ }^{\text {a, d, * }}$ \\ ${ }^{a}$ Institute of Materials Science and Nanotechnology, National Nanotechnology Research Center (UNAM), Bilkent University, Ankara, 06800, Turkey \\ ${ }^{\mathrm{b}}$ Life Sciences Application and Research Center, Gazi University, Ankara, 06830, Turkey \\ ${ }^{c}$ Department of Medical Biology and Genetics, Faculty of Medicine, Gazi University, Ankara, 06500, Turkey \\ ${ }^{\mathrm{d}}$ Neuroscience Graduate Program, Bilkent University, Ankara, 06800, Turkey \\ e Institute for Molecular Engineering, University of Chicago, Chicago, IL, 60637, USA
}

\section{A R T I C L E I N F O}

\section{Article history:}

Received 16 February 2017

Received in revised form 20 April 2017

Accepted 21 April 2017

Available online 22 April 2017

\section{Keywords:}

Peptide nanofiber

Burn injury

Heparin

Hydrogel

Self-assembly

\begin{abstract}
A B S T R A C T
Burn injuries are one of the most common types of trauma worldwide, and their unique physiology requires the development of specialized therapeutic materials for their treatment. Here, we report the use of synthetic, functional and biodegradable peptide nanofiber gels for the improved healing of burn wounds to alleviate the progressive loss of tissue function at the post-burn wound site. These bioactive nanofiber gels form scaffolds that recapitulate the structure and function of the native extracellular matrix through signaling peptide epitopes, which can trigger angiogenesis through their affinity to basic growth factors. In this study, the angiogenesis-promoting properties of the bioactive scaffolds were utilized for the treatment of a thermal burn model. Following the excision of necrotic tissue, bioactive gels and control solutions were applied topically onto the wound area. The wound healing process was evaluated at 7,14 and 21 days following injury through histological observations, immunostaining and marker RNA/protein analysis. Bioactive peptide nanofiber-treated burn wounds formed well-organized and collagen-rich granulation tissue layers, produced a greater density of newly formed blood vessels, and exhibited increased re-epithelialization and skin appendage development with minimal crust formation, while non-bioactive peptide nanofibers and the commercial wound dressing $3 \mathrm{M}^{\mathrm{TM}}$ Tegaderm ${ }^{\mathrm{TM}}$ did not exhibit significant efficiency over sucrose controls. Overall, the heparin-mimetic peptide nanofiber gels increased the rate of repair of burn injuries and can be used as an effective means of facilitating wound healing.
\end{abstract}

๑) 2017 Elsevier Ltd. All rights reserved.

\section{Introduction}

Burn injuries are the fourth most common type of trauma worldwide, and third-degree burns constitute one of the most severe injuries of the skin. Although recent developments in postinjury care have greatly improved the survival and recovery rates of patients suffering from severe burns, the considerable length of

\footnotetext{
* Corresponding author. Institute of Materials Science and Nanotechnology, Neuroscience Graduate Program, National Nanotechnology Research Center (UNAM), Bilkent University, Ankara, 06800, Turkey.

** Corresponding author. Institute for Molecular Engineering, University of Chicago, Chicago, IL, 60637, USA.

E-mail addresses: mguler@uchicago.edu (M.O. Guler), atekinay@bilkent.edu.tr (A.B. Tekinay).
}

the recovery process and the concurrent risk of infection contribute to patient mortality in burn injuries [23]. Even under ideal conditions, the formation of scar tissue follows wound closure, and may compromise the appearance and functionality of the healed tissue [12]. In addition, burn wounds are subject to a higher risk of necrosis compared to lacerations and blunt trauma injuries due to the oxidative and inflammatory stresses exerted by the zone of coagulation $[25,30]$. Consequently, untreated burn injuries often experience a period of progression where viable tissues adjacent to necrotic regions are themselves converted into necrotic tissue, increasing the depth and diameter of the injury [26,31]. Severe burn injuries are also associated with a rapid and systemic immune response that may result in septic shock due to a combination of increased capillary permeability, an excess of proteins in the interstitial space, and the overexpression of pro-inflammatory 
factors by macrophages [6]. Taken together, these factors necessitate the development of novel techniques that specifically address the unique pathophysiological needs of burn injuries, especially in the context of halting burn wound progression and facilitating tissue repair with minimal scar formation [29].

Hydrogels are effective materials for the treatment of burn injuries due to their water-retaining properties, which allow them to modulate the fluid balance at the site of injury and provide an environment similar to that present in native skin tissue. They can be functionalized with bioactive or antimicrobial agents to prevent secondary infections or accelerate the wound repair process [12]. Self-assembled peptide nanofiber gels contain bioactive sequences that effectively replicate the functions of many extracellular matrix proteins, allowing their use for the repair of a broad range of tissue injuries $[10,22,24]$. However, the function of peptide nanofiber systems depends strongly on their sequence, with minimal alterations resulting in major changes in toxicity, bioactivity and assembly kinetics [17,37]. Previously, peptide gels bearing the RGDlike RADA16 and ILVAGK/LIVAGK sequences were used as wound dressings for burn and laceration injuries [11,15], and we recently reported that heparin-mimetic peptide nanofibers (HM-PA, lauryl$\operatorname{VVAGEGD(K-psb)S-Am)~increase~re-epithelialization~and~granula-~}$ tion tissue formation in a rat acute skin wound model [35]. In contrast, a non-bioactive control gel (bearing a similar secondary structure but no heparin-mimetic motifs) provided no improvement over sucrose-treated controls, suggesting that the sequence (rather than the presence of the gel as a scaffold) was effective in promoting wound healing. HM-PA was shown to stimulate angiogenesis in vivo and exhibit a strong affinity to the growth factors VEGF, HGF, and FGF-2 in vitro, which is related to its ability to enhance wound repair [13]. However, post-injury progression of tissue necrosis, local accumulation of inflammatory cytokines, rapid fluid loss from blood vessels at the site of injury and an extended period of inflammation complicate the recovery of burn injuries, limiting the applicability of wound healing scaffolds in their treatment [34]. Consequently, it is interesting to use same peptide system for promotion of wound recovery under the unique microenvironmental conditions associated with burn wounds.

In the present study, we show that HM-PA nanofiber gels facilitate the recovery of burn injuries caused by a thermal burn model developed for the evaluation of topical treatment methods. Wound regeneration was monitored through histological observations, immunostaining and marker protein expressions across 21 days of recovery, and blood vessel densities, collagen organization and skin appendage formation were quantified to determine the effect of bioactive PA treatment on the repair process. HM-PA treatment was observed to enhance angiogenic activity during early regeneration; increase wound closure, re-epithelialization and granulation tissue formation rates, and promote the formation of skin appendages compared to non-bioactive peptide nanofibers, sucrose and $3 \mathrm{M}^{\mathrm{TM}}$ Tegaderm ${ }^{\mathrm{TM}}$ wound filler, suggesting that the HM-PA gel can be utilized as a potential platform for the treatment of burn wounds in addition to laceration injuries.

\section{Materials and methods}

\subsection{Materials}

9-Fluorenylmethoxycarbonyl (Fmoc) and tert-butoxycarbonyl (Boc) protected amino acids, lauric acid, 4-(2',4'-dimethoxymethyl-Fmoc-aminomethyl)-phenoxyacetamido-norleucylMBHA resin (Rink amide MBHA resin), Fmoc-Asp(OtBu)-Wang resin, 2-(1H-benzotriazol-1-yl)-1,1,3,3-tetramethyluronium hexafluorophosphate (HBTU) and diisopropylethylamine (DIEA) were purchased from NovaBiochem (Merck) and all other chemicals are purchased from Sigma-Aldrich, Thermo scientific, Invitrogen and Fisher. Antibodies used in this study were purchased from Millipore and Abcam.

\subsection{Synthesis and purification of molecules and gel formation}

An Fmoc solid phase peptide synthesis method was followed for the synthesis of peptide amphiphile (PA) molecules. Two different solid supports were used for peptide synthesis: The positivelycharged K-PA (lauryl-VVAGK-Am) and negatively-charged HM-PA (Lauryl-VVAGEGD(K-psb)S-Am) were fabricated using Rink amide MBHA resin, while the other negatively-charged PA (E-PA (laurylVVAGE)) was synthesized on Fmoc-Glu(OtBu)-Wang resin. Synthesis protocols were identical to these provided by Uzunalli et al. [35]. The mass and purity of molecules were assessed with liquid chromatography-mass spectroscopy (LC-MS). LC-MS data were obtained with an Agilent LC-MS equipped with Agilent 6530 Q-TOF with ESI source and Zorbax SB-C8 $4.6 \mathrm{~mm} \times 100 \mathrm{~mm}$ column for acidic conditions and a Zorbax Extend-C18 $2.1 \times 50 \mathrm{~mm}$ column for basic conditions. Mobile phases of each column were a gradient of water and acetonitrile containing $0.1 \%$ formic acid (for basic peptides) or $0.1 \% \mathrm{NH}_{4} \mathrm{OH}$ (for acidic peptides). Residual TFA adhering to positively charged PAs was removed by treatment with $\mathrm{HCl}$ solution, and the remaining peptide residue was lyophilized. An Agilent 1200 series preparative reverse-phase high performance liquid chromatography (HPLC) system was used for the purification of molecules and was equipped with a Zorbax Extend-C18 $21.2 \times 150 \mathrm{~mm}$ column for basic conditions and a Zorbax SB-C8 $21.2 \times 150 \mathrm{~mm}$ column for acidic conditions. As the mobile phases of each column, a gradient of water and acetonitrile containing $0.1 \%$ TFA or $0.1 \% \mathrm{NH}_{4} \mathrm{OH}$ was used according to charge of conditions.

In order to enable nanofibrous gel formation through charge neutralization, each oppositely-charged PA was reconstituted in $0.25 \mathrm{M}$ sucrose solution prepared in ultrapure water and mixed at the molar ratio of 1:2 for the HM-PA/K-PA combination (resulting in a bioactive PA mixture) and 1:1 $\mathrm{M}$ ratio for the E-PA/K-PA (resulting in a non-bioactive control PA mixture). Total charges of both mixtures were -1 . The $\mathrm{pH}$ of peptide solutions was adjusted to 7.4 and peptide mixtures were sterilized with UV light for $1 \mathrm{~h}$ prior to use.

\subsection{Physical and mechanical characterization of self-assembled peptide nanofiber networks}

\subsubsection{Scanning electron microscopy (SEM)}

Nanofiber networks formed by HM-PA/K-PA (1:2 M ratio) and EPA/K-PA (1:1 M ratio) were inspected through SEM. Samples for SEM were prepared by incubating peptide nanofiber gels (1 wt \%) with a final volume $60 \mu \mathrm{L}$ for $20 \mathrm{~min}$ on silicon wafers. The peptide nanofiber gels were then dehydrated by sequential treatment with increasing concentrations of ethanol $(20 \%, 40 \%, 60 \%, 80 \% \mathrm{v} / \mathrm{v})$ for 2 min per wash and left in $100 \%$ absolute ethanol until drying in a critical point drier (Tousimis, Autosamdri-815B). Before the imaging process, samples were coated with $6 \mathrm{~nm}$ of $\mathrm{Au} / \mathrm{Pd}$ layer and images were taken with a FEI Quanta 200 FEG scanning electron microscope under high vacuum with $5 \mathrm{kV}$ beam energy.

\subsubsection{Oscillatory rheology}

An Anton Paar Physica RM301 rheometer operating with a $25 \mathrm{~mm}$ parallel plate at $21{ }^{\circ} \mathrm{C}$ was used for oscillatory rheology measurements. In order to measure storage $\left(G^{\prime}\right)$ and loss $\left(G^{\prime \prime}\right)$ moduli, HM-PA or control nanofiber gels (1 wt \%) with total volume of $300 \mu \mathrm{L}$ were loaded on the center of the lower plate and incubated for 15 min prior to measurement while upper plate was brought to a $0.5 \mathrm{~mm}$ gap position. While taking storage moduli $\left(\mathrm{G}^{\prime}\right)$ and loss moduli $\left(G^{\prime \prime}\right)$ measurements, angular frequency was 
adjusted 100 to $0.1 \mathrm{rad} / \mathrm{s}$, with a shear strain of $0.5 \%$. The process was repeated 3 times for each peptide nanofiber gel and consecutive measurements were averaged for each sample.

\subsubsection{Circular dichroism (CD)}

Secondary structures of HM-PA nanofiber or control nanofiber assemblies were examined by $\mathrm{CD}$ spectroscopy using a J-815 Jasco J815 CD spectrophotometer at room temperature. Initially, PA stock solutions were prepared at $1 \mathrm{mM}$ concentration and diluted to a concentration of $3.6 \times 10^{-4} \mathrm{M}$ for CD spectroscopy. Measurements were obtained 3 times in the range of $300 \mathrm{~nm}-190 \mathrm{~nm}$ with $100 \mathrm{~nm} / \mathrm{min}$ scanning speed, $1 \mathrm{~nm}$ bandwidth and $0.1 \mathrm{~nm}$ data pitch and $0.1 \mathrm{~nm}$ data interval, with digital integration time (1 DIT) as $1 \mathrm{~s}$ at standard sensitivity. Three consecutive measurements were averaged for each sample. The results were modified and shown as molar ellipticity.

\subsection{In vivo burn model}

All procedures performed in the present study were approved by The Animal Experiments Ethical Committee of Gazi University. In vivo experiments were carried out with 3-4 month old (20-30 g) male BALB/c mice. Full-thickness dorsal burn wounds were created following a protocol modified from Sun et al. (Fig. 1) [32]. A total of 60 male mice were randomly divided into 4 groups for HM-PA nanofiber treatment $(\mathrm{n}=15)$, control nanofiber treatment $(n=15)$, sucrose solution treatment $(n=15)$ and $3 \mathrm{M}^{\mathrm{TM}}$ Tegaderm ${ }^{\mathrm{TM}}$ hydrogel treatment $(\mathrm{n}=15)$. Mice were maintained on ad libitum access to a standard laboratory diet in a 12-12 h light-dark cycle. Mice were anesthetized by intraperitoneal injection of ketamine hydrochloride $(45 \mathrm{mg} / \mathrm{kg}$ ) and xylazine hydrochloride $(5 \mathrm{mg} / \mathrm{kg}$ ). Under deep anesthesia, long hairs found on their dorsa were removed with a clipper and then shaved by a razor blade for complete hair removal. After the sterilization of underlying skin with povidone iodide, 3rd degree burns were generated with a custom made $200 \mathrm{~g}$ aluminum rod heated to $100{ }^{\circ} \mathrm{C}$ in a boiling water bath for $10 \mathrm{~min}$. The tip of the aluminum rod was used as a template to generate $1 \mathrm{~cm}^{2}$ wounds with square shapes on the posterior-dorsum of each mouse for $6 \mathrm{~s}$. Within 30 min of burning, mice were resuscitated through intraperitoneal injection of saline using half-strength Parkland formula ( $4 \mathrm{~mL} / \mathrm{kg}$ x percent body area). After $48 \mathrm{~h}$, full thickness skin layers were removed from the wounds using a biopsy punch to generate $6 \mathrm{~mm}$-diameter wounds. The debridement process was performed to accurately reflect the current medical standard for the treatment of burn injuries. The wounds were then treated with bioactive or non-bioactive peptide solutions, $0.25 \mathrm{M}$ sucrose solution or $3 \mathrm{M}^{\mathrm{TM}}$ Tegaderm ${ }^{\mathrm{TM}}$ hydrogel. Lyophilized peptide masses were dissolved in $0.25 \mathrm{M}$ sucrose solution to preserve osmotic balance. Gels were prepared by mixing oppositely charged PA molecules at a final concentration of $1 \mathrm{wt} \%$ immediately prior to application. $0.25 \mathrm{M}$ sucrose solution was used as a negative control, while $3 \mathrm{M}^{\mathrm{TM}}$ Tegaderm ${ }^{\mathrm{TM}}$ hydrogel was used as positive control. Following the application of each treatment, wounds were covered with gauze dressing and impregnated with saline, and a long-term adhesive, air permeable, elastic and sterile bandage (OctaCare ${ }^{\circledR}$ ) was placed over the wound to stabilize the position of treatments. Gels (and sucrose solution) were re-applied and the wounds were re-dressed daily for two days post-wounding. Mice were then individually caged and

A

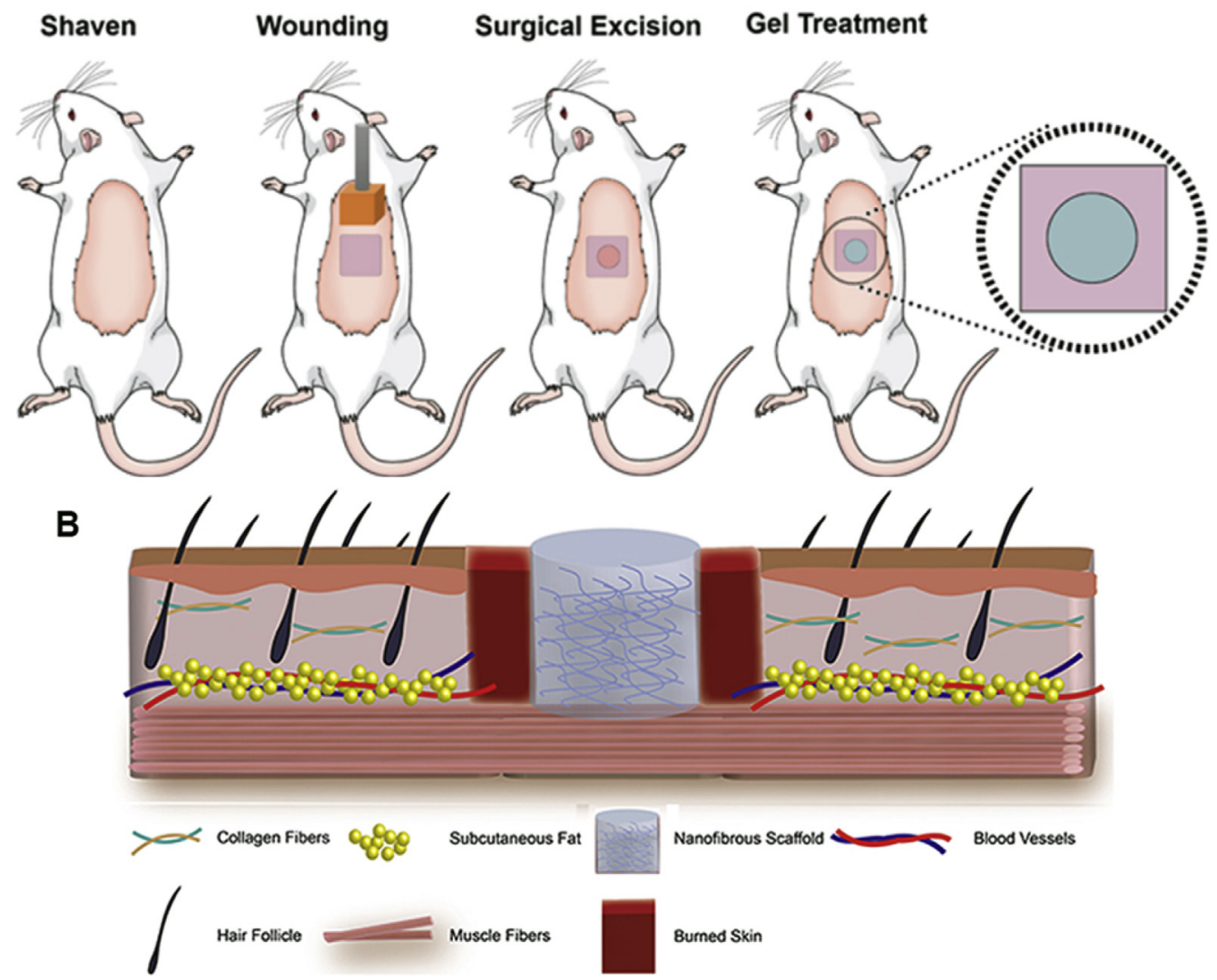

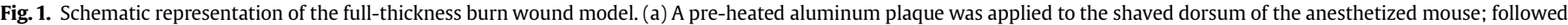

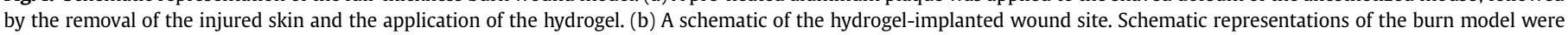
prepared using Mind the Graph software. 
allowed to eat and drink ad libitum and routine controls were conducted throughout the study for infection and body weight loss. Animals were sacrificed at each experimental end point (day 0 , day 7 , day 14, day 21) and their tissues were extracted to continue with further analysis. Half of the tissue was used for histological analysis and the other half was used for RNA and protein analysis.

\subsection{Wound area measurement}

Wounds were photographed on days 7, 9, 12, 14, 16, 19 and 21 post wounding with a ruler. The scab areas on the top of the wound were measured and calculated with Image J software (NIH, USA).

\subsection{Histological analysis}

Half of the skin tissues of sacrificed mice were collected at day 7, 14 and 21, and fixed in 10\% formalin. Following the fixation of extracts, samples were dehydrated in serially increasing concentrations of ethanol (70\%-100\%), embedded in paraffin and $5 \mu \mathrm{m}$ skin sections were serially taken using a microtome. Tissue sections were incubated at $58{ }^{\circ} \mathrm{C}$ overnight and stained with hematoxylin and eosin (H\&E), Masson's trichrome and picrosirius red with standard protocols after deparaffinization in xylene and rehydration in gradually decreasing concentrations of ethanol (\%100-\%70). All the quantifications from H\&E and Massons' trichrome staining were performed using Image J. Images from these stainings were acquired using an upright microscope (Zeiss Axio Scope A1) at $5 \times$ and $10 \times$ magnifications, and then merged to observe whole skin tissue. Firstly, whole granulation area was traced and measured from wound edges and remaining muscle tissue at both sites. Wound closure was measured at three different layers and their average was taken to best represent the extent of repair. The crust area was also traced and measured as an indicator of tissue necrosis following injury. Reepithelization was quantified by measuring the remaining gap between left and right wound edges. Newly formed skin appendages were counted in the burned area and their density was calculated. Newly synthesized collagen was also quantified. Orientation of collagen fibers was analyzed using the Image J plug-in Orientation J. Scoring was also confirmed by researchers who were blind to the study design.

\subsection{Immunohistochemical staining}

For immunohistological stainings, the tissue sections were deparaffinized in xylene and rehydrated in graded ethanol solutions and left in distilled water. Enzymatic antigen retrieval was performed with $0.1 \%$ pronase for $10 \mathrm{~min}$ at $35^{\circ} \mathrm{C}$ or heat mediated antigen retrieval was performed with sodium citrate buffer ( $\mathrm{pH}$ 6.0) using the guidelines provided by Abcam. Endogenous peroxidase activity was then inhibited with $3 \%$ hydrogen peroxide for $10 \mathrm{~min}$ at room temperature. Thereafter, normal goat serum was used to block non-specific binding sites by treating slides for $2 \mathrm{~h}$ at room temperature. Slides were incubated with anti-von Willebrand factor (1:400; ab6994, Abcam) overnight, followed by HRP conjugated goat anti-rabbit secondary antibody (1:500, Millipore (12-349)) on the next day. Antibody staining was followed by staining with 3,3'diaminobenzidine (DAB) and counterstaining with hematoxylin. Mounting was performed with a xylene based mounting medium. Digital images of the granulation area were acquired using an upright microscope (Zeiss Axio Scope A1) at $100 \times$ magnification and merged to count newly formed blood vessels. Quantifications were performed by Image granulation field.

\subsection{Protein and RNA isolation from tissue}

The simultaneous isolation of RNA and protein from burn wound samples was performed by using TRIzol reagent according to the manufacturer's protocol with minor adaptations and is briefly described below [3,7]. Firstly, tissue was weighed and cut into small pieces with the help of a sterile surgical knife. Then, tissue pieces were smashed with liquid nitrogen until they became powder-like, and samples were homogenized with a power homogenizer with $1 \mathrm{~mL}$ of TRIzol reagent per 50-100 mg of tissue sample. The homogenate was incubated for $5 \mathrm{~min}$ to permit the complete dissociation of the nucleo-protein complex, mixed with chloroform $(0.2 \mathrm{~mL}$ of chloroform per $1 \mathrm{~mL}$ of TRIzol reagent used for initial homogenization) with vigorous shaking and centrifuged, which yielded a colorless top aqueous phase, an interphase, and an organic bottom phase. RNA was precipitated from aqueous phase by addition of isopropanol $(0.5 \mathrm{~mL}$ of $100 \%$ isopropanol to aqueous phase per $1 \mathrm{~mL}$ of TRIzol reagent), mixed 15 times (up-down) and incubated at room temperature for $10 \mathrm{~min}$. Then, the mixture was centrifuged to allow the formation of a gel-like RNA pellet, washed with $2 \mathrm{~mL}$ of $75 \%$ ethanol, airdried for 5-10 min, dissolved in RNase-free water by pipetting and stored at $-20{ }^{\circ} \mathrm{C}$ until use. DNA was precipitated from the interphase and organic phase by the addition of ethanol $(0.3 \mathrm{~mL}$ of $100 \%$ ethanol per $1 \mathrm{~mL}$ TRIzol reagent used for initial homogenization) and centrifuged. Phenol-ethanol supernatant was removed and saved for protein precipitation by the addition of isopropanol (1.2 mL of isopropanol per $1 \mathrm{~mL}$ of TRIzol reagent was used for initial homogenization). The protein-isopropanol mixture was incubated overnight at $-20{ }^{\circ} \mathrm{C}$ to allow the precipitation of proteins and centrifuged to pellet them. The protein pellet was washed with $2 \mathrm{~mL}$ of cold $0.3 \mathrm{M}$ guanidine hydrochloride prepared in 95\% ethanol 3 times with 20 min of incubation between each washing, and then washed with ethanol at the last washing step. The protein pellet was left to air-dry for 5-10 min, dissolved with $10 \mathrm{M}$ urea containing a protease inhibitor cocktail (Sigma) [7] and stored at $-20{ }^{\circ} \mathrm{C}$ until use.

\subsection{Quantitative reverse transcription polymerase chain reaction (qRT-PCR)}

The gene expression profiles of angiogenesis inducing growth factors were evaluated by quantitative reverse transcription polymerase chain reaction (qRT-PCR) analysis. Yield and purity of extracted RNA were assessed using the Nanodrop 2000 system (Thermo Scientific). Primer sequences for growth factors were selected according to their suitability by using PREMIER Bio-soft software (Supplementary Table 1). Reaction efficiencies for each primer set were evaluated with standard curves using at least 5fold serial dilutions of total RNA after the optimization of their annealing temperatures. SuperScript III Platinum SYBR Green OneStep qRT-PCR kit (Invitrogen) was used for cDNA synthesis and qRTPCR according to the manufacturers' instructions. Reaction conditions were briefly as follows: $55^{\circ} \mathrm{C}$ for $5 \mathrm{~min}, 95^{\circ} \mathrm{C}$ for $5 \mathrm{~min}, 40$ cycles of $95^{\circ} \mathrm{C}$ for $15 \mathrm{~s}, T_{m}\left(60{ }^{\circ} \mathrm{C}, 60^{\circ} \mathrm{C}\right.$ and $58^{\circ} \mathrm{C}$ for VEGF, bFGF, GAPDH, respectively) for $30 \mathrm{~s}$, and $40{ }^{\circ} \mathrm{C}$ for $1 \mathrm{~min}$, which was followed by melting curve analysis to confirm product specificity after each reaction. VEGF and bFGF gene expression profiles were analyzed at days 7,14 , and 21 . For the analysis of expression, primary gene expression data were normalized to the expression level of GAPDH in each run, which served as the internal control gene. Each group was then normalized to the expression levels of sucrose solution treated groups, which served as a negative control. mRNA levels were calculated according to the comparative $\mathrm{Ct}$ method with efficiency correction for each target gene. 


\subsection{Western blot analysis}

After sacrificing the animals, half of the tissue samples collected from animals were taken into microcentrifuge tubes and immediately frozen with liquid nitrogen to further characterize the expression levels of proteins at days 7,14 and 21 of the burn wound healing process. Total protein in each sample was solubilized with $0.3 \mathrm{~mL}$ of $10 \mathrm{M}$ urea and quantified with BCA assays (Thermo Scientific) using a 1:50 dilution rate to eliminate the absorbance coming from urea itself and to accurately quantify the protein stock concentration. Equal amounts of proteins (70 ng, $20 \mu \mathrm{L}$ ) were resolved on $10 \%$ SDS-PAGE under denaturing and reducing conditions and transferred to polyvinylidene difluoride (PVDF) membranes (Thermo Scientific). Membranes were blocked with 5\% nonfat milk in TBS-T for $1 \mathrm{~h}$ at room temperature and then incubated with primary antibody at $4{ }^{\circ} \mathrm{C}$ overnight. Anti-VEGF and anti- $\alpha$ SMA antibodies were purchased from Millipore, ABS82, (1:750) and 04-1094, (1:1000), respectively. Anti-GAPDH antibody was used as internal control and purchased from Millipore, MAB374 (1:500). After washing in TBS-T, membranes were incubated with a corresponding HRP-coupled secondary antibody for $1 \mathrm{~h}$ at room temperature. Anti-rabbit IgG-HRP (purchased from Abcam) and antimouse IgG-HRP (purchased from Millipore, 12-349) were used at dilutions of $1: 2000$ and $1: 1000$, respectively. Signals were visualized using enhanced chemiluminescence analysis on a ChemiDoc ${ }^{\mathrm{TM}}$ Imaging System with Image Lab ${ }^{\mathrm{TM}}$ Software (Bio-Rad). Protein concentrations were quantified with Image software and intensities of VEGF and $\alpha$-SMA were normalized to GAPDH.

\subsection{Statistical analysis}

Statistical analyses were performed by using GraphPad Prism 6 software. One way ANOVA with Tukey's post-tests or two way ANOVA with Bonferroni post-tests was employed to determine the statistical differences between groups, where appropriate. Error bars indicate the standard error of the mean. The level of significance was set at $\mathrm{p}<0.05$.

\section{Results}

\subsection{Synthesis and characterization of PA molecules}

Heparin-mimetic (HM-PA), negatively charged E-PA and positively charged K-PA molecules were synthesized by a solid phase peptide synthesis method, purified by preparative HPLC, and their molecular weights were confirmed by MS analysis prior to their use (Fig. S1). The structure of the HM-PA molecule was designed to mimic that of heparin through the presentation of sulfonate, hydroxyl and carboxylate groups, while E-PA was used as a nonbioactive control peptide lacking reactive groups on its amino acid side chains (Fig. 2a) [35]. When mixed with the positivelycharged K-PA at neutral $\mathrm{pH}$, both peptide molecules selfassembled into fibrous networks that closely resemble the structure of the extracellular matrix (Fig. $2 \mathrm{~d}$ and e) and feature an abundance of $\beta$-sheets, while individual PA molecules did not selfassemble in the absence of a charge-neutralizing agent and exhibited random coil structures (Fig. 2b). Storage and loss moduli of both bioactive and non-bioactive peptide scaffolds were comparable to those of the commercial wound dressing $3 \mathrm{M}^{\mathrm{TM}}$ Tegaderm $^{\mathrm{TM}}$ (Fig. $2 \mathrm{~g}$ ). A representative image of PA nanofiber gels is shown in Fig. $2 \mathrm{f}$.

\subsection{Gel treatment and dorsal burn injury model}

Full-thickness burn wounds were visually monitored daily
(Fig. 3a, detailed view presented in Fig. S5) and 5 animals from each group were sacrificed for histological analysis at days 7, 14 and 21. Wound recovery was observed to be enhanced in wounds treated with HM-PA peptide nanofibers, with significantly smaller wound areas at days 12,14 , and 16 (Fig. 3b). In contrast, neither the non-bioactive peptide mixture nor the $3 \mathrm{M}^{\mathrm{TM}}$ Tegaderm ${ }^{\mathrm{TM}}$ filling enhanced wound closure compared to the negative control $(0.25 \mathrm{mM}$ sucrose $)$ within the first 16 days of recovery, although wound closure was complete in all groups at day 21. In addition, HM-PA peptide nanofiber-treated wounds exhibited a dense fur covering on top of the closed wound (which was shaved prior to wound imaging), while $3 \mathrm{M}^{\mathrm{TM}}$ Tegaderm ${ }^{\mathrm{TM}}$ - and sucrose-treated groups had limited fur growth (Fig. 3a).

\subsection{Histological analysis}

The effective recovery of burn wounds depends not only on the closure of the wound itself, but also on the replacement of damaged extracellular matrix with freshly deposited collagen, the removal of local debris from the wound site and the formation of new skin appendages [16]. As such, skin sections taken at 7, 14 and 21 days following peptide nanofiber gel, 3M $\mathrm{M}^{\mathrm{TM}}$ Tegaderm $^{\mathrm{TM}}$ or sucrose application were stained with H\&E and Masson's trichrome to observe the local morphology of the site of injury (Fig. 4a). Granulation, re-epithelialization, crust formation, wound contraction and skin appendage development were also monitored to determine the extent of functional wound recovery; representative images denoting the quantified regions are provided in Fig. S7. The gradual decrease of granulation area is one of the main hallmarks of skin wound recovery and occurs through the contractile activity of myofibroblasts. HM-PA peptide nanofiber gel treatment was found to result in significantly smaller granulation tissue areas at day 7 , suggesting that the heparin-mimetic hydrogel enhanced fibroblast recruitment and/or the differentiation of local fibroblasts into myofibroblasts (Fig. 4b). A similar trend was also observed in average wound distance results, which also strongly depend on myofibroblast activity and suggest that wound contraction was enhanced by bioactive peptide nanofiber gel treatment at day 7 (Fig. 4c).

In addition to the physical contraction of the site of injury, wound recovery also depends on the local remodeling of the extracellular matrix for the removal of local debris and deposition of new matrix proteins. While HM-PA peptide nanofiber treatment had a significant impact on wound closure at the earlier stages of the recovery process, its effects on matrix remodeling were longer-lasting. In particular, bioactive peptide nanofiber treated wounds had significantly shorter gaps in newly formed epithelial tissue at both day 7 and day 14, exhibiting mean epithelial gap distances of 4.4 and $1.6 \mathrm{~mm}$ at these time periods (compared to 6.5 and $4.7 \mathrm{~mm}$ for sucrose and 7.3 and $4.5 \mathrm{~mm}$ for $3 \mathrm{M}^{\mathrm{TM}}$ Tegaderm ${ }^{\mathrm{TM}}$ ) (Fig. 4d). In addition, the successful remodeling of the wound site following HM-PA peptide nanofiber treatment was also associated with the rapid degradation of the wound crust, which was 9.7- and 9.5-folds thinner than the sucrose and $3 \mathrm{M}^{\mathrm{TM}}$ Tegaderm ${ }^{\mathrm{TM}}$-treated groups at day 14 (Fig. 4e). Furthermore, bioactive peptide nanofiber-treated wounds had $16.67 \pm 5.03$ skin appendages per granulation area at day 21 , compared to $5.08 \pm 0.82$ and $10.49 \pm 1.73$ in sucrose and $3 \mathrm{M}^{\mathrm{TM}}$ Tegaderm ${ }^{\mathrm{TM}}$-treated wounds, suggesting that extracellular matrix remodeling was mostly complete by this time period and that the remodeled skin was well into the process of being repopulated by skin appendages (Fig. 4f). 
A<smiles></smiles>

B

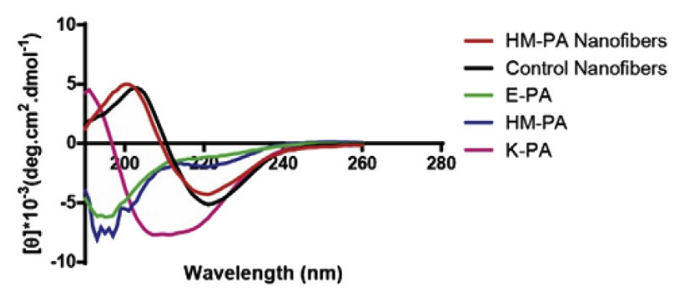

HM-PA (Lauryl-VVAGEGD(K-p-sulfobenzoate)S)-Am)

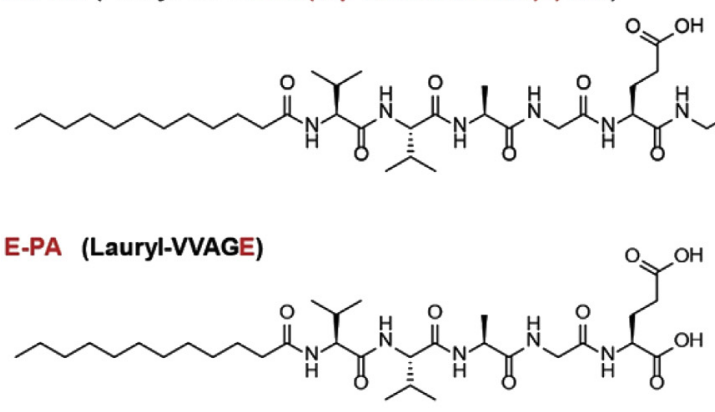

D

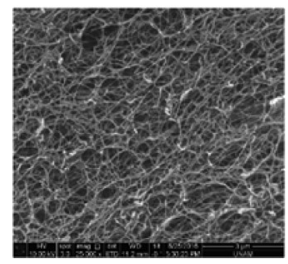

HM-PA Nanofibers
E

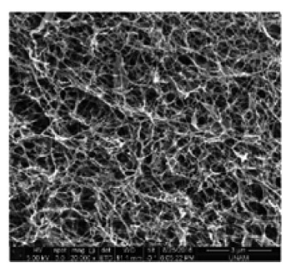

Control Nanofibers
$\mathbf{F}$

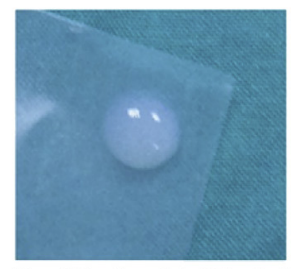

Peptide Amphiphile Gel
C

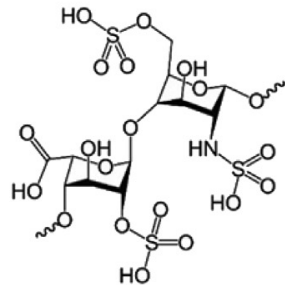

Heparin
$\mathbf{G}$

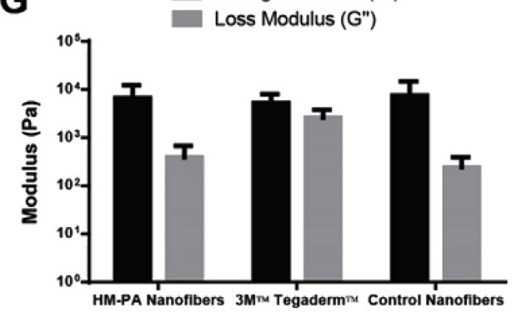

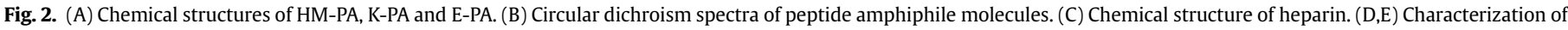
peptide nanofibers at pH 7.4 by SEM. (F) General appearance of peptide scaffold gels. (G) Rheology measurements of scaffolds. Scale bars are $5 \mu$ m.

\subsection{Modulation of angiogenesis and immunogenic response by} bioactive peptide nanofiber scaffolds

Bioactive peptide nanofiber treatment was observed to greatly enhance angiogenesis at the wound site at day 7 , with a smaller (but statistically significant compared to non-bioactive peptide nanofiber and $3 \mathrm{M}^{\mathrm{TM}}$ Tegaderm ${ }^{\mathrm{TM}}$ ) increase at day 14 , which is consistent with the early induction of angiogenesis in the presence of the bioactive peptide nanofibers (Fig. 5). However, compared to control nanofiber and $3 \mathrm{M}^{\mathrm{TM}}$ Tegaderm ${ }^{\mathrm{TM}}$, blood vessel density was significantly lower in HM-PA peptide nanofiber-treated wounds at day 21 , which suggests that wound recovery was complete at this time period and that most of the blood vessels present at the former wound site had been resorbed (Fig. 5B). The qRT-PCR analysis of VEGF and FGF-2 further supports these results, as the considerable increase in angiogenic factor expression at day 7 was observed to decrease at days 14 and 21 in the HM-PA peptide nanofiber-treated group (Fig. 6). The protein-level expression of $\alpha$ SMA was also significantly enhanced at day 7 in the HM-PA peptide nanofiber-treated group compared to control peptide nanofiber gel and sucrose solution, which may indicate the early differentiation of fibroblasts into myofibroblasts in the presence of the bioactive peptide nanofiber hydrogel. In addition to angiogenesis, the expression of immunogenic markers IL-4, IL1- $\beta$, IL-6, MCP-1 and TNF- $\alpha$ at day 7 also exhibited a downwards trend in HM-PA-treated wounds compared to other groups; however, only the difference between HM-PA and control nanofibers in IL-6 expression was statistically significant (Fig. S8).
A

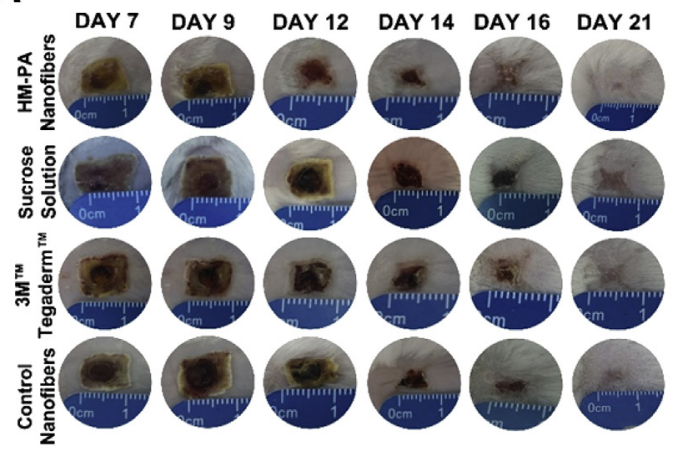

B

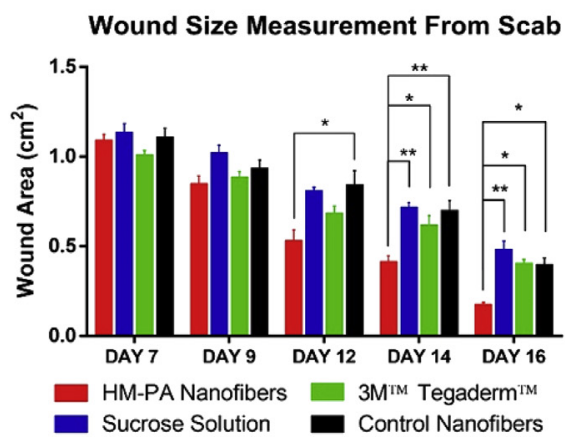

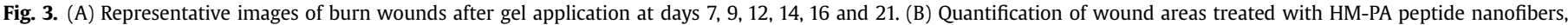

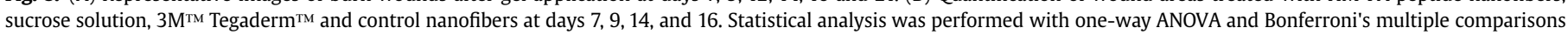
test. Error bars represent standard error of mean. 

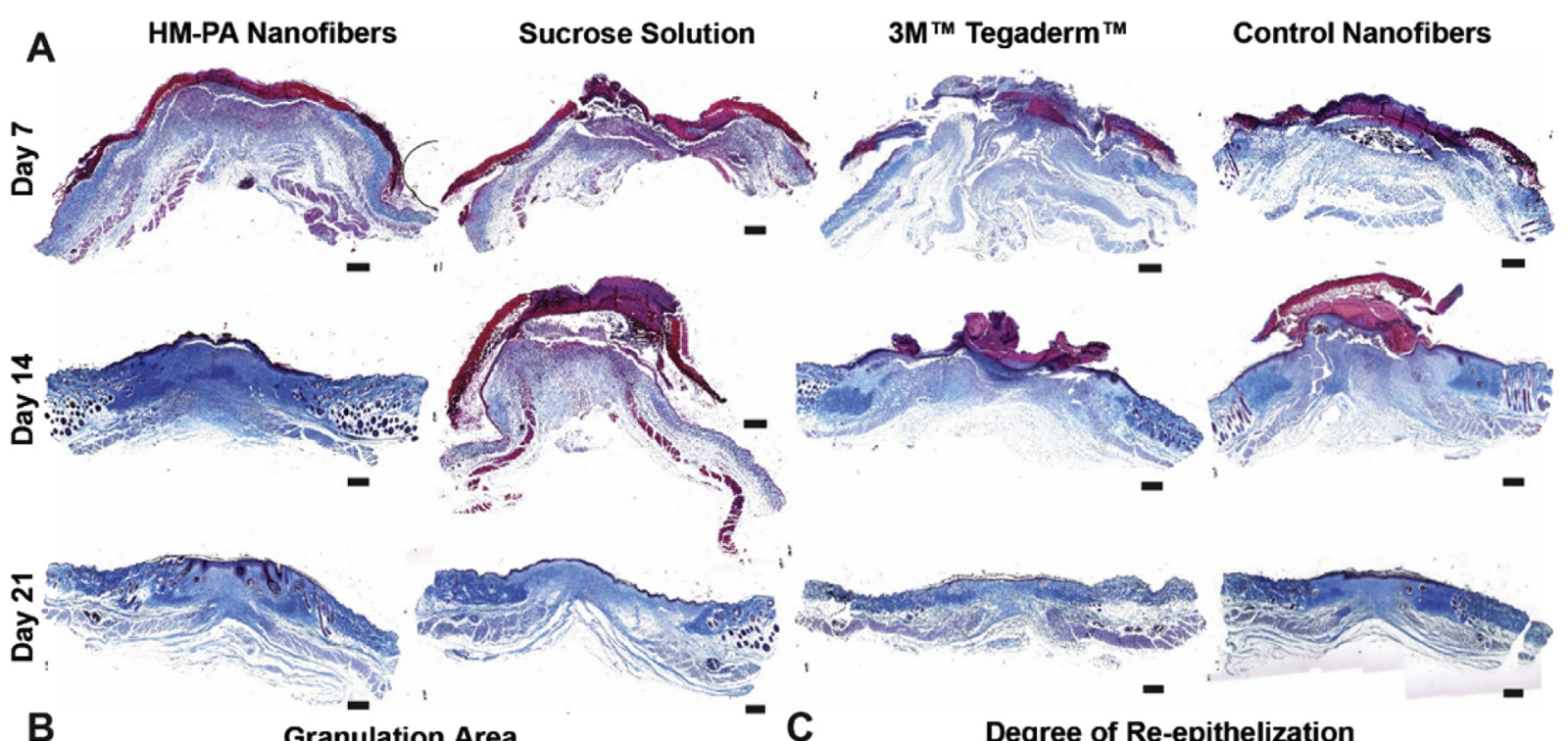

B

Granulation Area

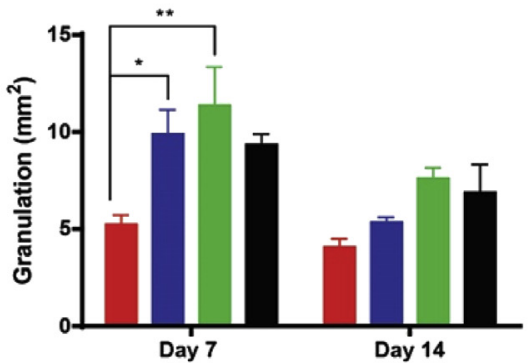

C

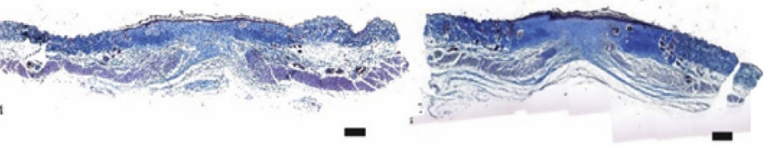

Degree of Re-epithelization

D

Crust Thickness

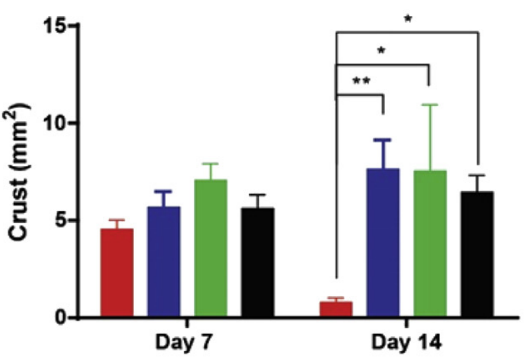

$\mathbf{F}$

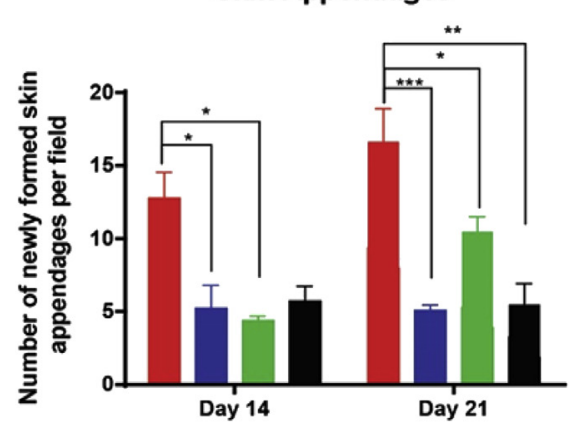

E

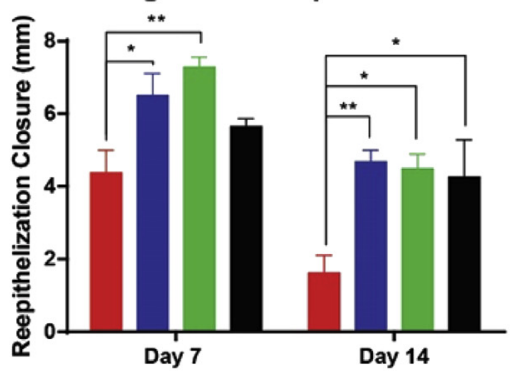

Average Wound Distance

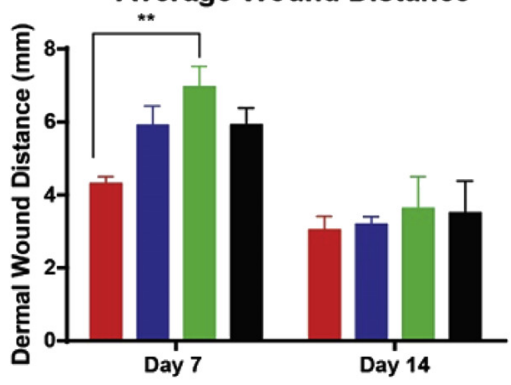

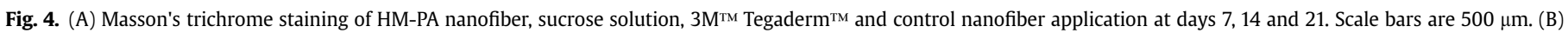

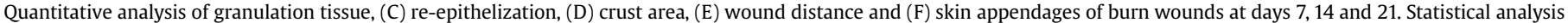
was performed with one-way ANOVA and Bonferroni's multiple comparisons test, ${ }^{*} \mathrm{p}<0.05$. Error bars represent standard error of mean.

\subsection{Collagen orientation}

The ability of bioactive and non-bioactive peptide nanofiber scaffolds and $3 \mathrm{M}^{\mathrm{TM}}$ Tegaderm ${ }^{\mathrm{TM}}$ to facilitate the deposition of wellcrosslinked collagen fibers was investigated through the picrosirius red staining of skin sections at day 21 (Fig. 7a). In healthy skin, picrosirius red dye stains in light/dark bands due to the presence of non-staining hair follicles, sebaceous and sweat glands, and a highly organized collagen structure (Fig. S9). In contrast, the wound area is characterized by uniform staining due to the absence of skin 


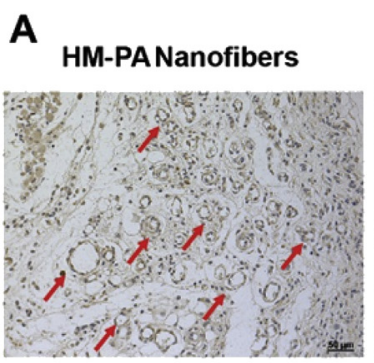

B

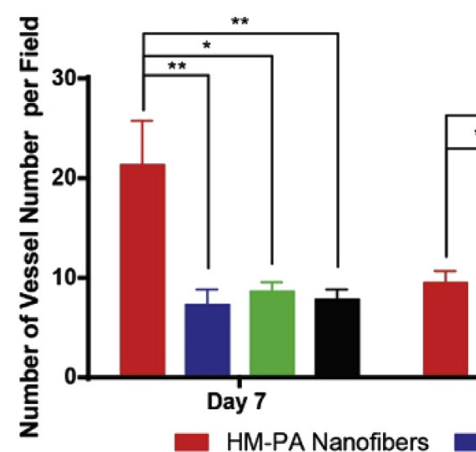

\section{DAY 7}

\section{Sucrose Solution}

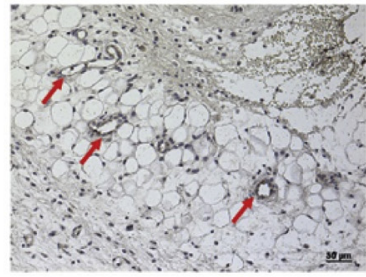

Neovascularization Degree
$3 M^{T M}$ Tegaderm ${ }^{T M}$

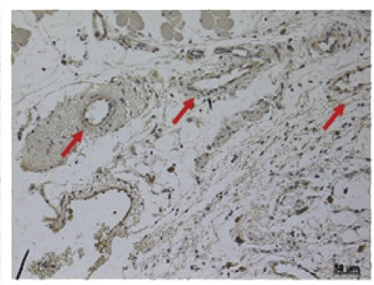

Control Nanofibers

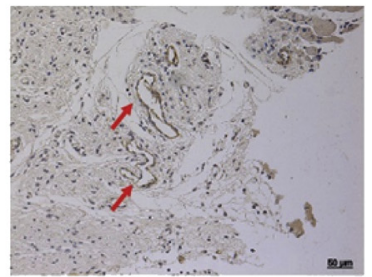

C

\section{Average Vessel Diameter}

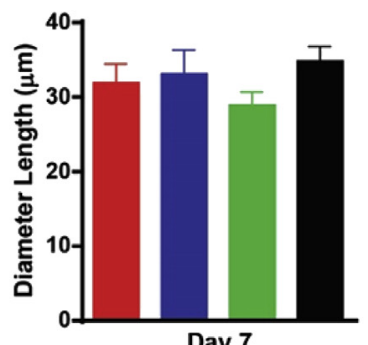

Day 7

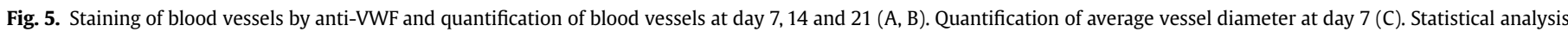

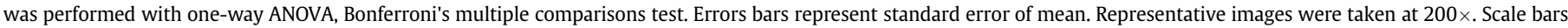
are $50 \mu \mathrm{m}$.

A

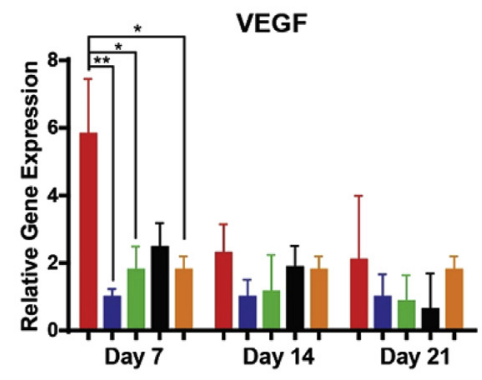

C

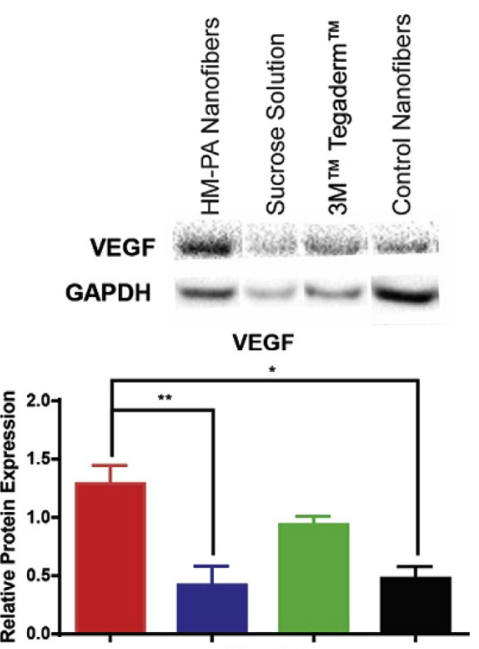

B

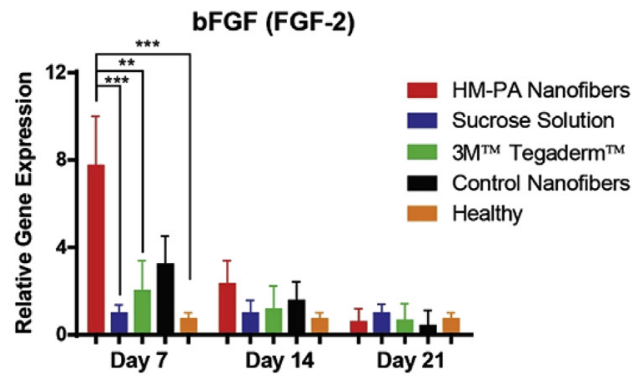

D
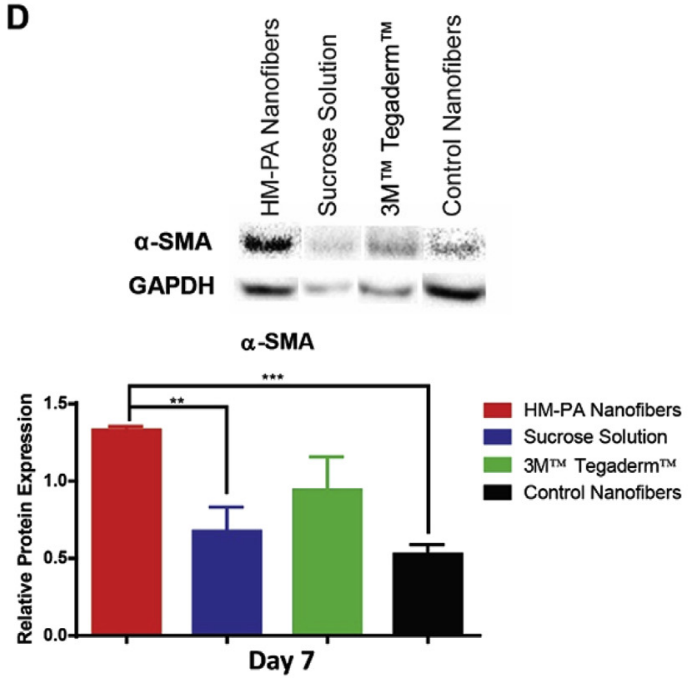

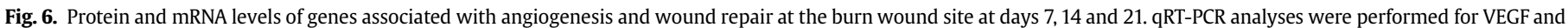

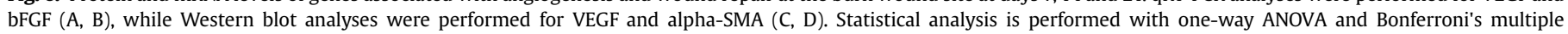
comparisons test. Error bars represent standard error of mean. 
appendages and disruption of the collagen network, and the restoration of the crosslinked collagen matrix is indicative of wound recovery. Accordingly, a highly homologous picrosirius red staining was observed in non-bioactive peptide nanofiber, $3 \mathrm{M}^{\mathrm{TM}}$ Tegaderm $^{\mathrm{TM}}$ and sucrose-treated burn injuries, suggesting that collagen crosslinking and skin appendage development are incomplete even at day 21 for these groups. However, a heterogeneous pattern of staining was observed in the HM-PA peptide nanofiber-treated wounds, which is consistent with an advanced state of recovery and the restoration of the regular architecture of skin. Bioactive hydrogel-treated wounds were also observed to exhibit two distinctive alignment directions under orientation analysis, which is similar to healthy skin and indicative of crosslinking (Fig. 7b and c). Non-bioactive peptide nanofiber-, 3M $3 \mathrm{M}^{\mathrm{TM}}$ Tegaderm ${ }^{\mathrm{TM}}$ - and sucrose-treated wounds, however, exhibited fiber orientation in only a single direction, suggesting that collagen crosslinking was not yet complete.

\section{Discussion}

Severe burn wounds are characterized by a strong local immune response, higher metabolic rates at the site of injury, increased cortisol and cytokine levels, ingress of fluid from extracellular space to the wound site and limited transport of oxygen and nutrients, which result in the gradual necrosis of affected tissue [8]. Consequently, the effective repair of burn wounds requires the modulation of metabolic, immune, homeostatic and angiogenic responses. Heparin and heparan sulfate are glycosaminoglycans that are abundantly expressed at the burn injury site and assist in the early wound healing process by preventing coagulation, activating a range of pro- and anti-inflammatory pathways and binding to growth factors such as VEGF, FGF, HGF and TGF- $\beta$ that stimulate the proliferation of fibroblasts, muscle cells, epithelial cells and endothelial cells [18]. However, heparin is rapidly degraded in biological environments and, due to its animal origin, poses a risk of zoonotic contamination, which has stimulated the development of more advanced delivery systems for replicating its effects on tissues [19]. Hydrogel scaffolds are especially useful for this purpose, as their similarities to the extracellular matrix, ease of modification, ability to constantly hydrate the site of injury and potential codelivery with cytokines, antibiotics, growth factors and/or stem cells are all highly relevant properties for the treatment of burn wounds [33].

HM-PA peptide nanofibers mimic the activity heparin and form a biocompatible gel at neutral $\mathrm{pH}$. The HM-PA peptide nanofibers were previously shown to bind growth factors, enhance osteochondrogenic differentiation, improve the success of pancreatic islet transplantation and stimulate the repair of normal and diabetic wound injuries $[1,13,28,36]$, and our present observations suggest that the growth factor-binding, water-retaining and glycosaminoglycan-mimicking features of HM-PA peptide nanofibers are also effective for stimulating the repair of full-thickness burn injuries. In particular, HM-PA peptide nanofiber-treated wounds had higher skin appendage formation, wound closure and re-epithelialization rates, as well as lower granulation tissue areas and thinner crust formation, at days 7 and 14 of the recovery
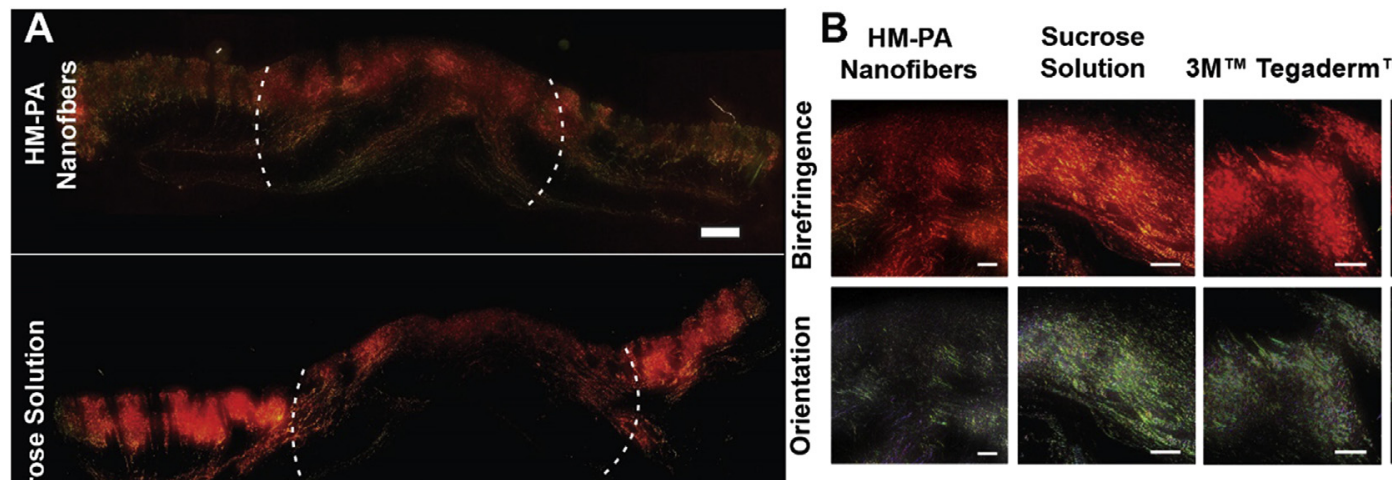

Contro

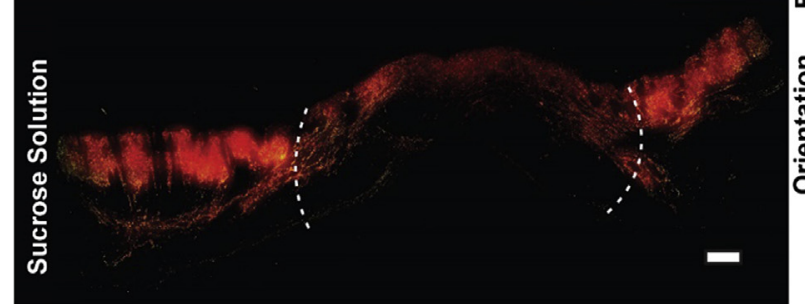

C

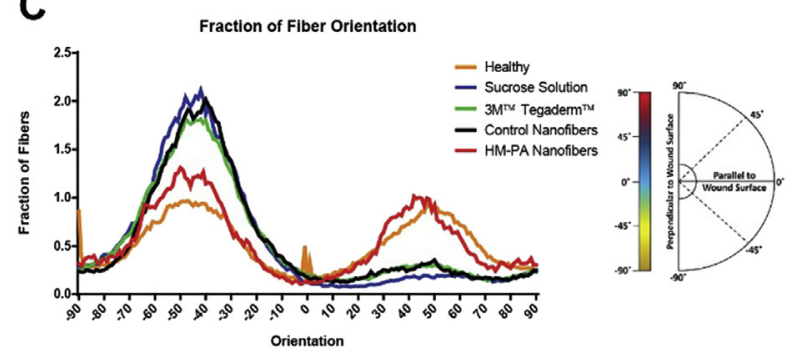

\section{Nanofibers}
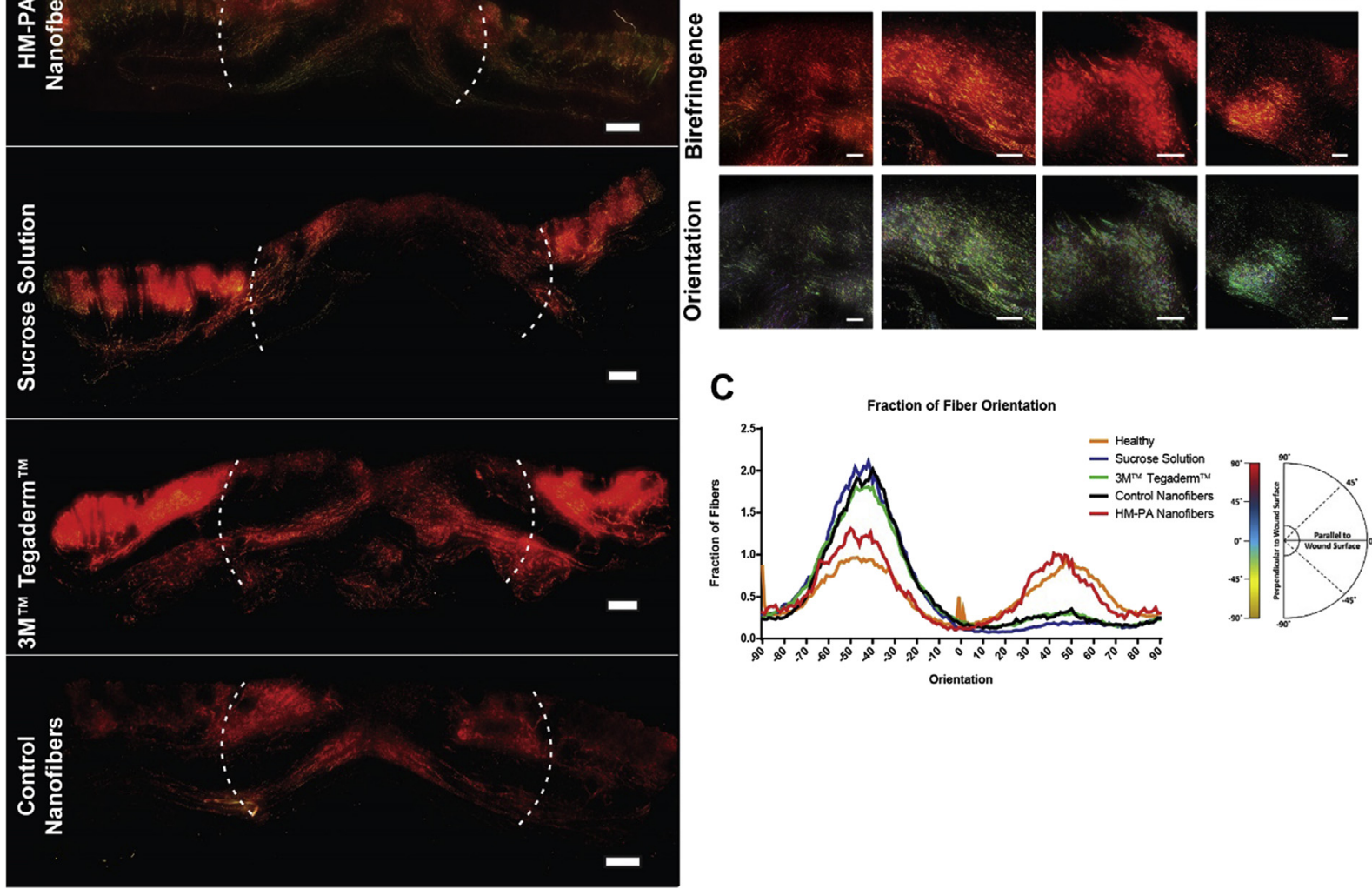

Fig. 7. Fiber directionality resembles healthy tissue in HM-PA peptide nanofiber-treated burn wounds. Picrosirius red staining was performed to visualize collagen fiber orientation and wound areas analyzed are highlighted with dashed lines (A, B). Healthy and HM-PA peptide nanofiber-treated fibers are distributed evenly between $-50^{\circ}$ and $+50^{\circ}$ orientations, while sucrose, $3 \mathrm{M}^{\mathrm{TM}}$ Tegaderm ${ }^{\mathrm{TM}}$ - and control nanofiber-treated wounds exhibit collagen orientation only in the $-50^{\circ}$ direction (C). (For interpretation of the references to colour in this figure legend, the reader is referred to the web version of this article.) 
process, which is consistent with the prominent roles played by heparin during early wound healing. Decreased crust formation and lower expressions of pro-immunogenic cytokines are especially relevant to the recovery of burn wounds, which are characterized by wound progression (i.e. expansion of necrotic regions, such as the wound crust, through post-injury damage) and a heightened inflammatory response [30]. While wound closure was mostly complete in all groups at day 21 , the bioactive peptide nanofiber-treated group nevertheless exhibited enhanced fur growth and a lack of scar tissue formation at later stages of recovery, and presented a greater amount of collagen remodeling in Masson's trichrome staining. Consequently, HM-PA treatment was able to support wound closure during the early repair process while minimizing scar formation by stimulating skin appendage formation and allowing the deposition of a well-crosslinked collagen matrix during the remodeling stage. In addition, one disadvantage associated with peptide hydrogels is that they degrade rapidly at the wound site, which we have counteracted here by applying each gel thrice at days 0,1 and 2. Gel re-application may be continued further into the proliferation stage to obtain more pronounced results at the end of the experimental period, and individual peptide nanofiber sequences can be tailored to each stage of recovery to optimally enhance the entire wound repair process.

Angiogenesis is another aspect of wound healing and plays an especially important role in burn injuries due to the anoxic and nutrient-poor environment that characterizes these wounds [33]. However, blood vessels are typically resorbed following the completion of the healing process, and excessive angiogenesis at later stages of wound healing is also detrimental for the functionality of the repaired tissue, potentially contributing to the development of hypertrophic scars [20]. Consequently, a balance between angiogenic and antiangiogenic responses is necessary for wound recovery, and the HM-PA peptide nanofibers are able to answer this need by mediating the required transition between blood vessel production and resorption, possibly through their gradual degradation at the site of injury. Accordingly, the presence of HM-PA during early wound repair is able to effectively stimulate angiogenesis (as shown by von Willebrand factor staining at days 7 and 14, as well as VEGF expression analysis at day 7), while its degradation during the following days would allow the remodeling step to proceed. As HM-PA peptide nanofibers have previously been shown to specifically interact with $\mathrm{VEGF}_{165}$ through its heparinbinding domain [13], it is likely that the promotion of angiogenesis occurs through the retention of endogenously produced VEGF, enhancement of its presentation, and/or stimulation of autocrine signaling from the cells at the site of injury, all of which are known roles of heparin. Promotion of cellular recruitment and differentiation is another possible mechanism behind enhanced angiogenesis and VEGF production, as heparin and its binding factors (such as EGF) have been reported to induce fibroblast recruitment, proliferation and differentiation $[4,5,9,14,27]$.

Following injury, the collagen matrix at the wound site is completely denatured, leaving a disordered and necrosispromoting extracellular environment. During the wound repair process, the cellular debris at the wound site is removed by inflammatory cells, and a new, well-crosslinked collagen matrix is deposited by local and recruited fibroblasts. Collagen alignment can therefore be used as a measure of the wound healing progress, as healthy extracellular matrix is characterized by two axes of collagen fibers that intersect to create a basket-woven appearance, while the old, dysfunctional extracellular matrix exhibits only a single collagen axis [2]. In sucrose and $3 \mathrm{M}^{\mathrm{TM}}$ Tegaderm ${ }^{\mathrm{TM}}$-treated wounds, orientation analysis showed that collagen fibers were arranged in only a single axis, while healthy and HM-PA peptide nanofiber-treated tissues were characterized by the deposition of collagen in two axes of orientation. This enhancement of collagen crosslinking was also supported by the early expression of $\alpha$-SMA, which is responsible for fibroblast activation, wound contraction and extracellular matrix remodeling. In addition, sucrose- and $3 \mathrm{M}^{\mathrm{TM}}$ Tegaderm ${ }^{\mathrm{TM}}$-treated samples exhibited some "gaps" without picrosirius red staining, suggesting that collagen deposition was incomplete in these samples, while the entire dermal layer was stained extensively with picrosirius red for healthy and HM-PA peptide nanofiber-treated samples. The ability of HM-PA to induce the deposition of a well-crosslinked collagen matrix appears to be an indirect consequence of its regeneration-promoting effects during earlier stages of recovery, as the material is subject to biodegradation and would be present only in minute quantities at the end of the 21-day experimental period.

\section{Conclusions}

In summary, we demonstrated that the heparin-mimetic peptide nanofibers are able to support the repair of full-thickness burn injuries by mediating wound contraction and re-epithelialization, preventing scar formation, and stimulating the development of skin appendages. In addition, enhanced wound recovery in the heparin-mimetic peptide nanofiber-treated group was found to be associated with the induction of neovascularization and the deposition of an extensively crosslinked collagen matrix. It is especially notable that the commercial wound filling $3 \mathrm{M}^{\mathrm{TM}}$ Tegaderm $^{\mathrm{TM}}$ was significantly less effective in many of these aspects. The burn injury and treatment model used in the present study closely resembles the standard of care for burn wounds, which entails the removal of necrotic tissue and the implantation of autografts and/or collagen-based scaffolds. However, the wounds were subcritical and accounted for c. $1.5 \%$ of the total body skin area of mice. In addition, human skin is associated with a sparsity of hair follicles, increased epidermal thickness and differences in immune cell populations compared to murine skin, which should be considered for the applicability of the HM-PA hydrogel to severe burns in human patients. Nevertheless, we suggest that the HM-PA peptide nanofiber system is suitable for adaptation to clinical settings for minor to moderate burn injuries that do not necessitate autograft treatment.

\section{Conflict of interest}

The authors declare no conflict of interest.

\section{Acknowledgement}

We thank Z. Erdogan for LC-MS analysis of peptide amphiphiles. We also would like to thank I. Ulusoy and M. Unal for their valuable help in animal care and N. Erbal for contributions in histology analyses. Fatih Yergoz was supported by TUBITAK BIDEB 2210-C graduate fellowship. This research did not receive any specific grant from funding agencies in the public, commercial, or not-for-profit sectors.

\section{Appendix A. Supplementary data}

Supplementary data related to this article can be found at http:// dx.doi.org/10.1016/j.biomaterials.2017.04.040.

\section{References}

[1] E. Arslan, M.O. Guler, A.B. Tekinay, Glycosaminoglycan-mimetic signals direct the osteo/chondrogenic differentiation of mesenchymal stem cells in a threedimensional peptide nanofiber extracellular matrix mimetic environment, 
Biomacromolecules 17 (2016) 1280-1291.

[2] S.A. Castleberry, A. Golberg, M.A. Sharkh, S. Khan, B.D. Almquist, W.G. Austen, M.L. Yarmush, P.T. Hammond, Nanolayered siRNA delivery platforms for local silencing of CTGF reduce cutaneous scar contraction in third-degree burns Biomaterials 95 (2016) 22-34.

[3] P. Chomczynski, A reagent for the single-step simultaneous isolation of RNA DNA and proteins from cell and tissue samples, Biotechniques 15 (1993) 532-534, 536-7.

[4] C. DeBlois, M.F. Côté, C.J. Doillon, Heparin-fibroblast growth factor-fibrin complex: in vitro and in vivo applications to collagen-based materials, Biomaterials 15 (1994) 665-672.

[5] A. Desmoulière, L. Rubbia-Brandt, G. Grau, G. Gabbiani, Heparin induces alphasmooth muscle actin expression in cultured fibroblasts and in granulation tissue myofibroblasts, Lab. Investig. 67 (1992) 716-726.

[6] J.A. Farina, M.J. Rosique, R.G. Rosique, Curbing inflammation in burn patients, Int. J. Inflam. 2013 (2013) 715645.

[7] A.B. Hummon, S.R. Lim, M.J. Difilippantonio, T. Ried, Isolation and solubilization of proteins after TRIzol extraction of RNA and DNA from patient materia following prolonged storage, Biotechniques 42 (2007) 467-470, 472.

[8] P.I. Jewo, I.O. Fadeyibi, Progress in burns research: a review of advances in burn pathophysiology, Ann. Burns Fire Disasters 28 (2015) 105-115.

[9] G. Kirkland, K. Paizis, L.L. Wu, M. Katerelos, D.A. Power, Heparin-binding EGFlike growth factor mRNA is upregulated in the peri-infarct region of the remnant kidney model: in vitro evidence suggests a regulatory role in myofibroblast transformation, J. Am. Soc. Nephrol. 9 (1998) 1464-1473.

[10] Y. Loo, M. Goktas, A.B. Tekinay, M.O. Guler, C.A. Hauser, A. Mitraki, Selfassembled proteins and peptides as scaffolds for tissue regeneration, Adv. Healthc. Mater. 4 (2015) 2557-2586.

[11] Y. Loo, Y.C. Wong, E.Z. Cai, C.H. Ang, A. Raju, A. Lakshmanan, A.G. Koh H.J. Zhou, T.C. Lim, S.M. Moochhala, C.A. Hauser, Ultrashort peptide nanofibrous hydrogels for the acceleration of healing of burn wounds, Biomaterials 35 (2014) 4805-4814.

[12] M. Madaghiele, C. Demitri, A. Sannino, L. Ambrosio, Polymeric hydrogels for burn wound care: advanced skin wound dressings and regenerative templates, Burns Trauma 2 (2014) 153-161.

[13] R. Mammadov, B. Mammadov, M. Guler, A. Tekinay, Growth factor binding on heparin mimetic peptide nanofibers, Biomacromolecules 13 (2012) $3311-3319$.

[14] P. Martin, Wound healing - aiming for perfect skin regeneration, Science 276 (1997) $75-81$.

[15] H. Meng, L. Chen, Z. Ye, S. Wang, X. Zhao, The effect of a self-assembling peptide nanofiber scaffold (peptide) when used as a wound dressing for the treatment of deep second degree burns in rats, J. Biomed. Mater Res. B Appl. Biomater. 89 (2009) 379-391.

[16] M.C. Miller, J. Nanchahal, Advances in the modulation of cutaneous wound healing and scarring, Bio Drugs 19 (2005) 363-381.

[17] T. Moyer, J. Finbloom, F. Chen, D. Toft, V. Cryns, S. Stupp, pH and amphiphilic structure direct supramolecular behavior in biofunctional assemblies, J. Am. Chem. Soc. 136 (2014) 14746-14752.

[18] P. Olczyk, Ł. Mencner, K. Komosinska-Vassev, Diverse roles of heparan sulfate and heparin in wound repair, Biomed. Res. Int. 2015 (2015) 549417.

[19] S.J. Paluck, T.H. Nguyen, H.D. Maynard, Heparin-mimicking polymers: synthesis and biological applications, Biomacromolecules 17 (11) (2016) 3417-3440.
[20] J.W. Penn, A.O. Grobbelaar, K.J. Rolfe, The role of the TGF- $\beta$ family in wound healing burns and scarring: a review, Int. J. Burns Trauma 2 (2012) 18-28.

[22] K. Rajangam, H.A. Behanna, M.J. Hui, X. Han, J.F. Hulvat, J.W. Lomasney, S.I. Stupp, Heparin binding nanostructures to promote growth of blood vessels, Nano Lett. 6 (2006) 2086-2090.

[23] M.P. Rowan, L.C. Cancio, E.A. Elster, D.M. Burmeister, L.F. Rose, S. Natesan, R.K. Chan, R.J. Christy, K.K. Chung, Burn wound healing and treatment: review and advancements, Crit. Care 19 (2015) 243.

[24] C.M. Rubert Pérez, N. Stephanopoulos, S. Sur, S.S. Lee, C. Newcomb, S.I. Stupp, The powerful functions of peptide-based bioactive matrices for regenerative medicine, Ann. Biomed. Eng. 43 (2015) 501-514.

[25] A.A. Salibian, A.T. Rosario, L. e. A. Severo, L. Nguyen, D.A. Banyard, J.D. Toranto, G.R. Evans, A.D. Widgerow, Current concepts on burn wound conversion-A review of recent advances in understanding the secondary progressions of burns, Burns 42 (2016) 1025-1035.

[26] D. Schmauss, F. Rezaeian, T. Finck, H.G. Machens, R. Wettstein, Y. Harder, Treatment of secondary burn wound progression in contact burns-a systematic review of experimental approaches, J. Burn Care Res. 36 (2015) e176-89.

[27] A. Schmitt-Gräff, A. Desmoulière, G. Gabbiani, Heterogeneity of myofibroblast phenotypic features: an example of fibroblastic cell plasticity, Virchows Arch. 425 (1994) 3-24.

[28] B. Senturk, M.O. Cubuk, M.C. Ozmen, B. Aydin, M.O. Guler, A.B. Tekinay, Inhibition of VEGF mediated corneal neovascularization by anti-angiogenic peptide nanofibers, Biomaterials 107 (2016) 124-132.

[29] P. Shakespeare, Burn wound healing and skin substitutes, Burns 27 (2001) $517-522$.

[30] J.W. Shupp, T.J. Nasabzadeh, D.S. Rosenthal, M.H. Jordan, P. Fidler, J.C. Jeng, A review of the local pathophysiologic bases of burn wound progression, J. Burn Care Res. 31 (2010) 849-873.

[31] V. Singh, L. Devgan, S. Bhat, S.M. Milner, The pathogenesis of burn wound conversion, Ann. Plast. Surg. 59 (2007) 109-115.

[32] G. Sun, Y.I. Shen, C.C. Ho, S. Kusuma, S. Gerecht, Functional groups affect physical and biological properties of dextran-based hydrogels, J. Biomed. Mater. Res. A 93 (2010) 1080-1090.

[33] G. Sun, X. Zhang, Y.I. Shen, R. Sebastian, L.E. Dickinson, K. Fox-Talbot, M. Reinblatt, C. Steenbergen, J.W. Harmon, S. Gerecht, Dextran hydrogel scaffolds enhance angiogenic responses and promote complete skin regeneration during burn wound healing, Proc. Natl. Acad. Sci. U. S. A. 108 (2011) 20976-20981.

[34] V.K. Tiwari, Burn wound: how it differs from other wounds? Indian J. Plast. Surg. 45 (2012) 364-373.

[35] G. Uzunalli, R. Mammadov, F. Yesildal, D. Alhan, S. Ozturk, T. Ozgurtas, M.O. Guler, A.B. Tekinay, Angiogenic heparin-mimetic peptide nanofiber gel improves regenerative healing of acute wounds, ACS Biomater. Sci. Eng. (2016), http://dx.doi.org/10.1021/acsbiomaterials.6b00165.

[36] G. Uzunalli, Y. Tumtas, T. Delibasi, O. Yasa, S. Mercan, M.O. Guler, A.B. Tekinay, Improving pancreatic islet in vitro functionality and transplantation efficiency by using heparin mimetic peptide nanofiber gels, Acta Biomater. 22 (2015) 8-18.

[37] M. Webber, J. Tongers, M. Renault, J. Roncalli, D. Losordo, S. Stupp, Development of bioactive peptide amphiphiles for therapeutic cell delivery, Acta Biomater. 6 (2010) 3-11. 